\title{
Holographic entanglement entropy of the BTZ black hole
}

\author{
Mariano Cadon:* and Maurizio Melis \\ Dipartimento di Fisica, Università di Cagliari, and INFN sezione di Cagliari, \\ Cittadella Universitaria 09042 Monserrato, ITALY
}

\begin{abstract}
We investigate quantum entanglement of gravitational configurations in 3D AdS gravity using the AdS/CFT correspondence. We derive explicit formulas for the holographic entanglement entropy (EE) of the BTZ black hole, conical singularities and regularized $\mathrm{AdS}_{3}$. The leading term in the large temperature expansion of the holographic EE of the BTZ black hole reproduces exactly its Bekenstein-Hawking entropy $S_{B H}$, whereas the subleading term behaves as $\ln S_{B H}$. We also show that the leading term of the holographic EE for the BTZ black hole can be obtained from the large temperature expansion of the partition function of a broad class of 2D CFTs on the torus. This result indicates that black hole $\mathrm{EE}$ is not a fundamental feature of the underlying theory of quantum gravity but emerges when the semiclassical notion of spacetime geometry is used to describe the black hole.
\end{abstract}

\section{INTRODUCTION}

At low energies any quantum theory of gravity must allow for the classical space-time description of general relativity. Low-energy gravity is a macroscopic phenomena that, at least to some extent, should be described without detailed knowledge of the fundamental microscopic theory that holds at Planckian scales. From this point of view a gravitational system is not very different from a condensed matter system, whose macroscopical behavior allows for an effective description in terms of low-energy degrees of freedom. A strong evidence that this may work also for gravity is represented by the microscopic interpretation of the black hole entropy: in a number of cases the BekensteinHawking black hole entropy could be reproduced as Gibbs entropy, without detailed information about the underlying microscopical description of quantum gravity degrees of freedom [1, 2, 3, 34, 5, 6, 7, 8, 9$]$.

A feature of many-body systems, which can be used to gain information about macroscopic collective effects, is quantum entanglement. It gives a measure of spatial correlations between parts of the system and it is measured by the entanglement entropy (EE). In the last years the notion of EE has been used with success as a tool for understanding quantum phases of matter, but its application to gravitational systems remains problematic [10, 11, 12, 13, 14, 15, 16, 17].

The semiclassical EE of quantum matter fields in a classical gravitational background (e.g. a black hole) is not universal (it depends on the number of matter fields species) and it is not clear if it can be extended to the quantum phase of gravity [18, [19, 20]. The very notion of EE for pure quantum gravity is not easy to define. The main obstruction comes from the fact that in the usual Euclidean quantum gravity formulation the metric, except its boundary value, cannot be fixed a priori (see e.g. Ref. [21]), whereas the usual, flat-space notion of EE requires to fix lengths in bulk spacelike regions [53].

A possible way out of these difficulties is to consider gravity theories with conformal field theory (CFT) duals (see e.g. [26]). The advantage of considering this kind of theories is twofold: 1) One can define the EE of a gravity configuration in terms of the EE of a field theory in which spacetime geometry is not dynamic; 2) At least for CFTs in two dimensions explicit and simple formulas for the EE are known [27, 28, 29]. The main drawback of this approach is related to the fact that the gravity/CFT correspondence is holographic (usually it takes the form of an AdS/CFT correspondence). Spatial correlations in the bulk gravity theory are codified in a highly nonlocal way in the correlations of the boundary CFT. This is particularly evident in the so-called UV/IR relation that relates large distances on AdS space with the short distances behavior of the boundary CFT [30, 31].

Because of this difficulty the AdS/CFT correspondence has been only partially fruitful for understanding the EE of gravitational configurations, in particular of black holes. Some progress in this direction has been achieved in the general case in Ref. [32, 33, 34] and for the 2D case in Ref. [24, 25, 35]. Strangely enough, the AdS/CFT correspondence has been used with much more success in the reversed way, i.e. to compute the EE of boundary CFTs in terms of bulk geometrical quantities [36, 37, 38, 39, 40, 41, 42].

\footnotetext{
*Electronic address: mariano.cadoni@ca.infn.it
}

${ }^{\dagger}$ Electronic address: maurizio.melis@ca.infn.it 
In this paper we will investigate quantum entanglement in the context of three-dimensional (3D) AdS gravity, in particular the Bañados-Teitelboim-Zanelli (BTZ) black hole, using the $\mathrm{AdS}_{3} / \mathrm{CFT}_{2}$ correspondence. We will tackle the problem using a standard method for studying correlations in QFT: we will introduce in the boundary 2D CFT two external length-scales, a thermal wavelength $\beta=1 / T$ ( $T$ is the temperature of the CFT) and a spatial length $\gamma$ which is the measure of the observable spatial region of our $2 \mathrm{D}$ universe. Varying $\beta$ we can probe thermal correlations of the CFT at different energy scales, whereas varying $\gamma$ we can probe the spatial correlations at different length scales.

We will show that the AdS/CFT correspondence, and in particular the UV/IR relation, will allow us to identify in natural way $\beta$ and $\gamma$ in terms of the two fundamental bulk length scales, the horizon of the BTZ black hole $r_{+}$and the AdS length $L$. This will allow us, using well-known formulas for the EE of 2D CFTs and modular symmetry, to associate an "holographic" EE to regularized $\mathrm{AdS}_{3}$, the BTZ black hole and $\mathrm{AdS}_{3}$ with conical singularities. We will also show that the leading term in the EE of the BTZ black hole can be obtained in terms of the large temperature expansion of the partition function of a broad class of CFTs on the torus. This strongly supports the intrinsic semiclassical nature of the black hole EE.

The structure of the paper is as follows. We will briefly review some well-known facts about the EE for 2D CFTs in Sect. II and about $\mathrm{AdS}_{3}$ gravity and the $\mathrm{AdS}_{3} / \mathrm{CFT}_{2}$ correspondence in Sect. III. In Sect. IV we will discuss the modular invariance of the dual boundary CFTs on the torus. In Sect. V we will investigate the relevance of UV/IR relation for the calculation of the EE. In sections VI, VII, VIII we will use our approach to derive the holographic EE of, respectively, regularized $\mathrm{AdS}_{3}$, the BTZ black hole and $\mathrm{AdS}_{3}$ with conical singularities. In section IX we will compare the holographic EE for the BTZ black hole with the large temperature asymptotic expansion of the thermal entropy of most common 2D CFTs on the torus. Finally, in Sect. X we will present our concluding remarks.

\section{ENTANGLEMENT ENTROPY OF 2D CFT}

Most of the progress in understanding EE in QFT has been achieved in the case of a 2D CFT. This is because the conformal symmetry can be used to determine the form of the correlation functions of the theory [27, 28, 29].

Let us consider a 2D spacetime with a compact spacelike dimension of length $\Sigma$ and with $S^{1}$ topology. When only a spacelike slice $Q$ (of length $\gamma$ ) of our universe is accessible for measurement, we loose information about the degrees of freedom (DOF) localized outside in the complementary region $P$ and we have to trace over these DOF. The entanglement entropy originated by tracing over the unobservable DOF is given by the von Neumann entropy $S_{\text {ent }}=-\operatorname{Tr}_{Q} \hat{\rho}_{Q} \ln \hat{\rho}_{Q}$. The reduced density matrix $\hat{\rho}_{Q}=\operatorname{Tr}_{P} \hat{\rho}$ is obtained by tracing the density matrix $\hat{\rho}$ over states in the region $P$.

The resulting EE for the ground state of the 2D CFT is given by [27, 28, 29]

$$
S_{\text {ent }}^{(\mathcal{C})}=\frac{c+\bar{c}}{6} \ln \left(\frac{\Sigma}{\epsilon \pi} \sin \frac{\pi \gamma}{\Sigma}\right)
$$

where $c$ and $\bar{c}$ are the central charges of the 2D CFT and $\epsilon$ is an ultraviolet cutoff necessary to regularize the divergence originated by the presence of a sharp boundary separating the region $P$ from the region $Q$. Thus, Eq. (1D) gives the EE for a CFT at zero temperature and with a spacelike dimension with $S^{1}$ topology, i.e. for a 2D CFT on a cylinder $\mathcal{C}$, whose timelike direction is infinite.

For $\Sigma \gg \gamma$ the compact spacelike dimension becomes also infinite and the EE is independent of $\Sigma$. Eq. (1) gives the EE for a 2D CFT at zero temperature on the plane $\mathcal{P}$ : [27, 28, 29]

$$
S_{\text {ent }}^{(\mathcal{P})}=\frac{c+\bar{c}}{6} \ln \left(\frac{\gamma}{\epsilon}\right) .
$$

We can also consider a 2D CFT at finite temperature $T=1 / \beta$ and a noncompact spacelike dimension. When only a spacelike slice of length $\gamma$ is accessible to measurement, the EE turns out to be that of a 2D CFT on a cylinder $C$, whose spacelike direction is infinite [28]:

$$
S_{\text {ent }}^{(C)}=\frac{c+\bar{c}}{6} \ln \left(\frac{\beta}{\epsilon \pi} \sinh \frac{\pi \gamma}{\beta}\right) .
$$

It is important to stress that the cylinder $C$ can be obtained as the limiting case of a torus $\mathcal{T}(\beta, \gamma)$ with cycles of length $\beta$, $\gamma$, when $\gamma \gg \beta$. In Sect. IX we will use this feature to relate the thermal entropy of a CFT on a torus with the EE of a CFT on the cylinder $C$. 


\section{III. $\mathrm{ADS}_{3}$ GRAVITY AND $\mathrm{ADS}_{3} / \mathrm{CFT}_{2}$ CORRESPONDENCE}

The EE of a QFT gives information about the spatial correlations of the theory. It follows that the EE of a 2D CFT, which is the holographical dual of 3D gravity, should contain information about bulk quantum gravity correlations. The most important example in this context is given by the correspondence between 3D AdS gravity and 2D CFT $\left(\mathrm{AdS}_{3} / \mathrm{CFT}_{2}\right)$. Classical, pure $\mathrm{AdS}_{3}$ gravity is described by the action

$$
A=\frac{1}{16 \pi G_{3}} \int d^{3} x\left(R+\frac{2}{L^{2}}\right),
$$

where $L$ is the de Sitter length and $G_{3}$ is 3D Newton constant. The exact form of the 2D CFT dual to 3D AdS gravity still remains a controversial point [43, 44, 45]. However, in the large $N$ (central charge $c \gg 1$ ) regime, i.e. in region of validity of the gravity description, we know that the dual CFT has central charge [46]

$$
c=\bar{c}=\frac{3 L}{2 G_{3}} .
$$

$\mathrm{AdS}_{3}$ classical gravity allows for three kinds of configurations. These solutions of the action (4) can be classified in terms of orbits (elliptic, hyperbolic, parabolic) of the $S L(2, R)$ group manifold [43, 47, 48]. The solutions corresponding to elliptic orbits can be written as

$$
d s^{2}=-\frac{1}{L^{2}}\left(r^{2}+r_{+}^{2}\right) d t^{2}+\left(r^{2}+r_{+}^{2}\right)^{-1} L^{2} d r^{2}+\frac{r^{2}}{L^{2}} d \phi,
$$

where $0 \leq t \leq \beta, 0 \leq \phi \leq 2 \pi L, 0 \leq r<\infty$ and $r_{+}$is a constant. The corresponding 3D Euclidean space has a contractible cycle in the spatial, $\phi$-direction. For generic values of $r_{+}$we have therefore a conical singularity in this direction. Only for $r_{+}=L$ the conical singularity disappears and the manifold becomes nonsingular 3D AdS space at finite temperature $1 / \beta$. The conformal boundary of the $3 \mathrm{D}$ spacetime is a torus with cycles of length $\beta$ and $2 \pi L$. Correspondingly, the dual CFT will live in the torus $\mathcal{T}(\beta, 2 \pi L)$. The CFT on the cylinder $\mathcal{C}$ discussed in Sect. II can be obtained in the limit $\beta \gg L$. This corresponds to consider $-\infty<t<\infty$ and $0 \leq \phi \leq 2 \pi L$.

The classical solutions of $3 \mathrm{D}$ gravity corresponding to hyperbolic orbits of $S L(2, R)$ are

$$
d s^{2}=-\frac{1}{L^{2}}\left(r^{2}-r_{+}^{2}\right) d t^{2}+\left(r^{2}-r_{+}^{2}\right)^{-1} L^{2} d r^{2}+\frac{r^{2}}{L^{2}} d \phi^{2}
$$

Now the 3D Euclidean manifold has a contractible cycle in the $t$-direction. For generic values of $\beta$ and $r_{+}$we have therefore a conical singularity in this direction. Only for $\beta=\beta_{H}$, where $\beta_{H}$ is the inverse Hawking temperature

$$
\beta_{H}=\frac{1}{T_{H}}=\frac{2 \pi L^{2}}{r_{+}}
$$

the conical singularity can be removed and the space describes the Euclidean BTZ black hole. The black hole has horizon radius $r_{+}$, and mass and (thermal) Bekenstein-Hawking (BH) entropy given by

$$
M=\frac{r_{+}^{2}}{8 G_{3} L^{2}}, \quad S_{B H}=\frac{\mathcal{A}}{4 G_{3}}=\frac{\pi r_{+}}{2 G_{3}} .
$$

Also in this case the conformal boundary of the $3 \mathrm{D}$ spacetime is the torus with cycles of length $\beta_{H}, 2 \pi L$ and the dual CFT will live on $\mathcal{T}\left(\beta_{H}, 2 \pi L\right)$. The CFT on the cylinder $C$ discussed in Sect. II can be obtained in the limit $L \gg \beta_{H}$. This corresponds to consider a CFT at finite temperature, $0 \leq t \leq \beta_{H}$, with noncompact spacelike dimension $-\infty<\phi<\infty$. In terms of the 3D bulk theory this corresponds to a macroscopical black hole with $r_{+} \gg L$.

The separating element between the two classes of solutions above corresponds to parabolic orbits of $S L(2, R)$,

$$
d s^{2}=-\frac{1}{L^{2}} r^{2} d t^{2}+\frac{L^{2}}{r^{2}} d r^{2}+\frac{r^{2}}{L^{2}} d \phi^{2}, \quad-\infty<t<\infty .
$$

The solution can be seen as the $r_{+}=0$ ground state of the BTZ black hole, i.e. the $M=0, T_{H}=0$ solution.

For $r_{+} \neq L$ the solution (6) has a conical singularity not shielded by an event horizon [47, 48]. The conical singularity can also be thought of as originated by a pointlike source of mass $m$. In the spectrum of AdS $\mathrm{A}_{3}$ gravity these solutions are located between the NS vacuum, $r_{+}=L$, and the RR vacuum, $r_{+}=0$. Therefore we will consistently take $0 \leq r_{+} \leq L$. 
Let us now briefly discuss the physical meaning of the conical singularity spacetime (6). To this end, let us rescale the coordinates in Eq. (6):

$$
r \rightarrow \frac{r_{+}}{L} r, \quad t \rightarrow \frac{L}{r_{+}} t, \quad \phi \rightarrow \frac{L}{r_{+}} \phi
$$

The metric becomes

$$
d s^{2}=-\left(\frac{r^{2}}{L^{2}}+1\right) d t^{2}+\left(\frac{r^{2}}{L^{2}}+1\right)^{-1} d r^{2}+\frac{r^{2}}{L^{2}} d \phi
$$

The previous expression describes thermal $\mathrm{AdS}_{3}$ in global coordinates but, owing to the rescaling of the coordinates we have now $0 \leq \phi \leq 2 \pi \Gamma L$, with $\Gamma=r_{+} / L$. The spacetime has a conical singularity originated by a deficit angle $2 \pi(1-\Gamma)=2 \pi\left(1-r_{+} / L\right)=2 \pi\left(1-2 \pi L / \beta_{\text {con }}\right)$, where we have introduced

$$
\beta_{\text {con }}=2 \pi L^{2} / r_{+},
$$

as the analogous of the inverse Hawking temperature $\beta_{H}$ to characterize the conical singularity. In the case of solution (7), setting $\beta=\beta_{H}$ eliminates the conical singularity, whereas for solution (6) we get a regular manifold (AdS $\mathrm{A}_{3}$ at finite temperature) for $\beta_{\text {con }}=2 \pi L$.

The conical singularity we have whenever $\beta_{c o n} \neq 2 \pi L$ represents the geometric distortion generated by a pointlike particle of mass $m=(1-\Gamma) / 4 G_{3}$. In order to find the holographic EE of the solution (6), (17) and (10), we have to discuss first the modular symmetry of the 2D CFT dual to 3D AdS gravity.

\section{MODULAR INVARIANCE}

It is well known that the partition function of a 2D CFT on the complex torus has to be invariant for transformation of the modular group $\operatorname{PSL}(2, Z)$

$$
\tau \rightarrow \frac{a \tau+b}{c \tau+d}
$$

where $a, b, c, d$ are integers satisfying $a d-b c=1, \tau=\omega_{2} / \omega_{1}$ is the modular parameter of the torus and $\omega_{1,2}$ are the periods of the torus. For simplicity we will take $\omega_{1}=\Sigma$ real and $\omega_{2}=i \beta$ purely imaginary. We are mainly interested in the modular transformation of the torus

$$
\tau \rightarrow-\frac{1}{\tau}
$$

3D spaces which are asymptotically AdS are locally equivalent. The asymptotic form of the coordinate transformations mapping the various spaces can be used to map one into the other the tori describing the associated conformal boundaries. For our discussion the relevant elements are the Euclidean BTZ black hole at Hawking temperature $1 / \beta_{H}, \mathrm{AdS}_{3}$ space with deficit angle $2 \pi\left(1-2 \pi L / \beta_{\text {con }}\right)$ and $\mathrm{AdS}_{3}$ at finite temperature $1 / \beta_{H}$. It will turn out that boundary tori associated with these three spaces are related by modular transformations of the torus.

Let us briefly review the well-known duality between the BTZ black hole and $\mathrm{AdS}_{3}$ at finite temperature [26, 49 ]. To this purpose, we use the fact that the Euclidean BTZ solution (17) with periodicity $t \sim t+\beta_{H}, \phi \sim \phi+2 \pi L$ can be mapped by a diffeomorphism into $\mathrm{AdS}_{3}$ in Poincaré coordinates

$$
d s^{2}=\frac{1}{x^{2}}\left(d y^{2}+d z d \bar{z}\right)
$$

where $z$ is a complex coordinate.

In the asymptotic $r \rightarrow \infty(x \rightarrow 0)$ region the map between the BTZ black hole and $\mathrm{AdS}_{3}$ in Poincaré coordinates is

$$
z=\exp \left[\frac{2 \pi}{\beta_{H}}(\phi+i t)\right] .
$$

In order to have a natural periodicity, we introduce a new complex variable $w$

$$
z=\exp (-2 \pi i w)
$$


so that $w=(-t+i \phi) / \beta_{H}$. One can now easily realize that the asymptotic conformal boundary of the BTZ black hole is a complex torus with metric $d s^{2}=d w d \bar{w}$. The periodicity of the imaginary $\left(\omega_{2}\right)$ and real $\left(\omega_{1}\right)$ part of $w$ are determined by the periodicity of $t, \phi: \omega_{2}=2 \pi i L / \beta_{H}, \quad \omega_{1}=1$. The modular parameter $\tau_{B T Z}=\omega_{2} / \omega_{1}$ of the torus is therefore

$$
\tau_{B T Z}=\frac{2 \pi i L}{\beta_{H}} .
$$

Consider now Euclidean $\mathrm{AdS}_{3}$ at finite temperature, described by the metric (12) with the periodicity $t \sim t+\beta_{H}$ and $\phi \sim \phi+2 \pi L$. The $r \rightarrow \infty$ asymptotic form of the map between $\mathrm{AdS}_{3}$ at finite temperature and $\mathrm{AdS}_{3}$ in Poincaré coordinates is

$$
z=\exp \frac{(t-i \phi)}{L}
$$

whereas the coordinate $w$ of Eq. (18) is now $w=\frac{1}{2 \pi L}(\phi+i t)$. The complex coordinate $w$ has now periodicity $\omega_{1}=1, \omega_{2}=i \beta_{H} / 2 \pi L$. The boundary of thermal $\mathrm{AdS}_{3}$ is a torus with modular parameter

$$
\tau_{A d S}=\frac{i \beta_{H}}{2 \pi L}
$$

Hence the boundary torus of the BTZ black hole and that of thermal $\mathrm{AdS}_{3}$ are related by the modular transformation

$$
\tau_{A d S}=-\frac{1}{\tau_{B T Z}} .
$$

Passing to consider the Euclidean solution with the conical singularity (6), we note that it is related to AdS 3 just by the rescaling (11). This changes the periodicity of the coordinates, which becomes $t \sim t+2 \pi L, \phi \sim \phi+4 \pi^{2} L^{2} / \beta_{\text {con }}$. Because the coordinate transformation mapping the boundary torus of conical singularity space into the boundary torus of $\mathrm{AdS}_{3}$ has the same form given by Eq. (20), it follows that the periodicity of the coordinate $w$ is now $\omega_{1}=2 \pi L / \beta_{\text {con }}, \omega_{2}=i$. If we set $\beta_{\text {con }}=\beta_{H}$ the periodicity of the two tori are related by

$$
\omega_{2}^{c o n}=\frac{i}{\omega_{1}^{A d S}}, \quad \omega_{1}^{c o n}=\frac{i}{\omega_{2}^{A d S}} .
$$

The boundary torus of Euclidean $\mathrm{AdS}_{3}$ with conical singularity characterized by the deficit angle $2 \pi\left(1-2 \pi L / \beta_{H}\right)$ has the same modular parameter as that of $\mathrm{AdS}_{3}$ at temperature $1 / \beta_{H}$. Notice that although the two manifolds have the same topology and the same boundary torus, they describe different three-geometries. The first is a singular one, whereas the latter is a perfectly well-behaved geometry. For this reason, one usually does not include $\mathrm{AdS}_{3}$ with conical singularities in the physical spectrum of the theory.

Because $\tau_{c o n}=\tau_{A d S}$, from Eq. (22) it follows immediately that, the boundary tori of AdS 3 with conical defect $2 \pi\left(1-2 \pi L / \beta_{H}\right)$ and that of the BTZ black hole at inverse temperature $\beta_{H}$ are related by the modular transformation

$$
\tau_{c o n}=-\frac{1}{\tau_{B T Z}} .
$$

\section{ENTANGLEMENT ENTROPY AND THE UV/IR RELATION}

As a consequence of the AdS/CFT correspondence the EE (11), (2) and (3) should give information about bulk quantum gravity correlators. More precisely, one would expect the EE in Eq. (11) to describe quantum correlations in the presence of conical singularity ([6) and the EE (3) of the thermal CFT to describe the interplay between thermal and quantum correlations in the black hole background (7). The main obstacle to make the above relation precise is due to the holographic nature of the AdS/CFT correspondence. Spatial correlations in the bulk gravity theory are codified in the boundary CFT in highly nonlocal way. Whereas the inverse temperature $\beta$ appearing in Eq. (3) can be naturally identified as the inverse of the black hole temperature (8), the same is not true for the parameters $\gamma$ and $\epsilon$ in Eqs. (11), (2) and (3).

Owing to the holographic nature of the correspondence, the bulk interpretation of these parameters requires careful investigation. The $\mathrm{AdS}_{p+1} / \mathrm{CFT}_{p}$ correspondence indicates a way to relate length scales on the boundary with length scales on the bulk, this is the UV/IR connection [30, 31]. Infrared effects in bulk, AdS $p+1$ gravity correspond to ultraviolet effects in the boundary $\mathrm{CFT}_{p}$ and vice versa. 
The UV/IR connection allows to identify the UV cutoff $\epsilon$ in Eq. (2) as an IR regulator of $\mathrm{AdS}_{3}$ gravity $\underline{30}$, 31. This can be done in the usual way by using the dilatation isometry of the metric (10) $r \rightarrow \lambda r, t \rightarrow \lambda^{-1} t, \phi \rightarrow \lambda^{-1} \phi$. Equivalently, one can introduce "cavity coordinates" on $\mathrm{AdS}_{3}$ and show that $\epsilon$ acts as infrared regulator of the "area" of the $S^{1}$ boundary sphere [30]. In fact, the regularized radius of the $\mathrm{S}^{1}$ is $R=L^{2} / \epsilon$. The same is true in terms of the coordinate $r$ parametrizing $\mathrm{AdS}_{3}$ in the modified Poincaré form (10): cutting off at length scale $<\epsilon$ the 2D CFT implies an infrared cutoff on the radial coordinate of $\mathrm{AdS}_{3}, r<\Lambda$, where [54]

$$
\Lambda=\frac{4 \pi^{2} L^{2}}{\epsilon} .
$$

The bulk interpretation of the parameter $\gamma$ in Eq. (2) is not as straightforward as that of $\epsilon . \gamma$ is not a simple external length scale we are using to cut off excitations of energy $<1 / \gamma$. It is the length of a localized spacelike slice of the $2 \mathrm{D}$ space on which the CFT lives. On the other hand, owing to the holographic, nonlocal nature of the bulk/boundary correspondence, we expect that any localization of DOF in the boundary will be lost by the correspondence with DOF on the bulk. If any localization property of the observable slice $Q$ is lost in the boundary/bulk duality, $\gamma$ can only play the role of an upper bound above which spatial correlations for the boundary CFT are traced out. Because of the UV/IR connection, on $\mathrm{AdS}_{3}$ this will correspond to tracing out the bulk DOF at small values of the radial coordinate $r$, i.e. for $r<\omega$, where

$$
\omega=\frac{4 \pi^{2} L^{2}}{\gamma} .
$$

It is important to stress that the bulk parameter $\omega$ has not the same physical meaning of the boundary parameter $\gamma$. Whereas $\gamma$ is the length of a spacelike slice, which is sharply separated from the observable region (hence it needs a UV regulator), $\omega$ has the much weaker meaning of a length scale below which spatial correlations are traced out. In particular in the $\mathrm{AdS}_{3}$ bulk there is no sharp boundary separating observable and unobservable regions.

In the next sections we will use this meaning of $\gamma$ and $\omega$ to interpret the EE (11), (2) and (3) as holographic entanglement entropies of gravitational configurations.

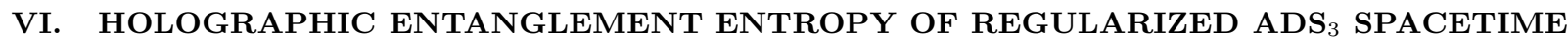

The AdS/CFT correspondence and the IR/UV connection described in the previous section allow us to give to the EE (2) a simple bulk interpretation: it is the EE of regularized AdS spacetime (10), i.e. it gives a measure of the von Neumann entropy that arises when an IR cutoff $\Lambda$ is introduced and quantum gravity correlations are traced out for $r<\omega$. Using Eqs. (25) and (26) into Eq. (21), we have $S_{\text {ent }}^{A d S}=\frac{c}{3} \ln \left(\frac{\Lambda}{\omega}\right)$ (we have used $\bar{c}=c$ ). The natural length scale for cutting off quantum bulk correlations is given by the AdS length $L: \omega=2 \pi L$. This means that we are considering curvature effects much smaller than $1 / L^{2}$. Using Eq. (26), this allows the identification of the boundary parameter in terms of the AdS length $L$

$$
\gamma=2 \pi L .
$$

The holographic EE of the regularized AdS spacetime

$$
S_{\text {ent }}^{A d S}=\frac{c}{3} \ln \left(\frac{\Lambda}{L}\right)
$$

has a simple geometric interpretation. Apart from a proportionality factor, it is the (regularized) proper length of the spacelike curve $t=$ const, $\phi=$ const. This can be easily shown integrating Eq. (10) for $L \leq r \leq \Lambda$.

\section{HOLOGRAPHIC ENTANGLEMENT ENTROPY OF THE BTZ BLACK HOLE}

The spinless BTZ black hole (7) can be considered as the thermalization at temperature $T=T_{H}$ of the AdS spacetime (10). On the 2D boundary of the AdS spacetime, and in the above discussed large temperature limit $r_{+} \gg L$, this thermalization corresponds to a plane/cylinder transformation that maps the CFT on the plane $\mathcal{P}$ in the CFT on the cylinder $C$. The conformal map plane/cylinder has the (Euclidean) form given in Eq. (17). One can easily check that the above transformation is the asymptotic form of the map between the BTZ black hole and $\mathrm{AdS}_{3}$ in Poincaré coordinates. The conformal transformation (17) maps the EE of a CFT on the plane $\mathcal{P}$ in the EE 
of a CFT in the cylinder $C$ [28], i.e. the EE of a CFT at zero temperature in a spacetime with noncompact spacelike dimension into the EE of a CFT at finite temperature. As a result, Eq. (2) is transformed in Eq. (3) with $\beta=\beta_{H}$. Correspondingly, the holographic EE of the regularized AdS spacetime becomes the holographic EE of the BTZ black hole

$$
S_{\text {ent }}^{B T Z}=S_{\text {ent }}^{C F T}\left(\gamma=2 \pi L, \beta=\beta_{H}\right)=\frac{c}{3} \ln \frac{2 L^{2}}{\epsilon r_{+}} \sinh \frac{\pi r_{+}}{L}
$$

The entanglement entropy (29) still depends on the UV cutoff $\epsilon$. A renormalized entropy $\tilde{S}_{\text {ent }}^{B T Z}$ can be defined by subtracting the contribution of the vacuum (the zero mass, zero temperature BTZ black hole solution). In terms of the dual CFT we have to subtract the entanglement entropy of the zero-temperature vacuum state. This is given by Eq. (2) with $\gamma=2 \pi L$. The renormalized entanglement entropy is therefore given by

$$
\tilde{S}_{e n t}^{B T Z}=S_{e n t}^{B T Z}-S_{e n t}^{v a c}=\frac{L}{2 G_{3}} \ln \frac{L}{\pi r_{+}} \sinh \frac{\pi r_{+}}{L} .
$$

As expected the renormalized entanglement entropy vanishes for $r_{+}=0$ (the BTZ black hole ground state).

The holographic entanglement entropy (30) for the BTZ black hole coincides exactly with the previously derived entropy for the 2D AdS black hole [24]. The 2D AdS black hole is the dimensional reduction of the spinless BTZ black hole. Using the relationship between $2 \mathrm{D}$ and $3 \mathrm{D}$ Newton constant $\Phi_{0}=L / 4 G_{3}$, Eq. (30) reproduces exactly the result of Ref. [24].

Macroscopic, i.e. large temperature, $r_{+} \gg L$, black holes correspond, in terms of the 2D CFT, to the thermal wavelength $\beta_{H}$ much smaller than the length $2 \pi L$. Expansion of Eq. (30) for $r_{+} / L \gg 1$ gives

$$
\tilde{S}_{B T Z}^{e n t}=\frac{\pi r_{+}}{2 G_{3}}-\frac{L}{2 G_{3}} \ln \frac{\pi r_{+}}{L}+O(1)=S_{B H}-\frac{L}{2 G_{3}} \ln S_{B H}+O(1) .
$$

The leading term in entanglement entropy is exactly the Bekenstein-Hawking entropy. This leading term describes the extreme situation in which thermal fluctuations dominates completely. In this limit the entanglement entropy is just a measure of thermodynamical entropy. The EE (the von Neumann entropy) for the CFT becomes extensive and it agrees with the Gibbs entropy of an isolated system of length $\gamma=2 \pi L$. The subleading term behaves as $\ln S_{B H}$ and describes the first corrections due to quantum entanglement.

The logarithmic correction in Eq. (31) matches exactly [55] the short scale correction for the quantum entropy of a scalar field in the BTZ Euclidean background found in Ref. [20]. The fact that a 3D bulk calculation of the quantum entropy give the same result of a boundary CFT calculation is not only a non trivial check of their correctness, but also a highly nontrivial check of the $\mathrm{AdS}_{3} / \mathrm{CFT}_{2}$ correspondence.

In principle, one could also consider the regime $\beta_{H} \sim 2 \pi L$ in which the full quantum nature of the entanglement entropy should be evident. However, this regime is singular from the black hole point of view: it corresponds to the $3 \mathrm{D}$ analogous of the Hawking-Page phase transition [50, 51].

It is interesting to notice that the identification $\gamma=2 \pi L$, which is crucial for deriving Eq. (29), can be obtained without using the UV/IR connection, just assuming that in the large $N$ limit the mass/temperature relationship for the BTZ black hole exactly reproduces that of a thermal 2D CFT.

From Eqs. (8), (9) one easily finds the mass-temperature relationship for the BTZ black hole,

$$
M=\frac{\pi^{2} L^{2}}{2 G_{3}} T_{H}^{2}
$$

On the other hand, in the large temperature limit $\gamma \gg \beta$ the entanglement entropy (3) reduces to the classical, extensive thermal entropy for an isolated system of length $\gamma$. The energy/temperature relationship for such a 2D $\mathrm{CFT}$ is given by ( $E_{0}$ is the energy of the vacuum)

$$
E-E_{0}=\frac{c}{12} \pi \gamma\left(T_{+}^{2}+T_{-}^{2}\right)
$$

where $T_{+}$and $T_{-}$are the temperatures for the right and left oscillators. Identifying the black hole mass $M$ with $E-E_{0}$ and the temperature $T_{H}=T_{+}=T_{-}$of the CFT thermal state with the Hawking temperature of the black hole, we easily find, comparing Eq. (33) with Eq. (32) and using Eq. (5), $\gamma=2 \pi L$. 


\section{A. Holographic Entanglement entropy of the rotating BTZ black hole}

The derivation of the EE for the spinless BTZ black hole can be easily extended to the rotating BTZ solution,

$$
d s^{2}=g(r) d t^{2}+g(r)^{-1} d r^{2}+r^{2}\left(\frac{d \phi}{L}-\frac{4 J G_{3}}{r} d t\right)^{2}, \quad g(r)=\frac{1}{r^{2} L^{2}}\left(r^{2}-r_{-}^{2}\right)\left(r^{2}-r_{+}^{2}\right),
$$

where $r_{ \pm}$are the positions of outer and inner horizons and $J$ is the black hole angular momentum. The thermodynamical parameters characterizing the black hole are the mass $M$, the angular momentum $J$, the Bekenstein-Hawking entropy $S_{B H}$, the temperature $T_{H}$ and the angular velocity $\Omega$ (acting as potential for $J$ ). These parameters satisfy the first principle $d M=T_{H} d S_{B H}+\Omega d J$ and can be written in terms of $r_{ \pm}$:

$$
\begin{aligned}
M & =\frac{r_{+}^{2}+r_{-}^{2}}{8 G_{3} L^{2}}, \quad J=\frac{r_{+} r_{-}}{4 G_{3} L}, \quad S_{B H}=\frac{\pi r_{+}}{2 G_{3}}, \\
T_{H} & =\frac{1}{2 \pi L^{2}}\left(\frac{r_{+}^{2}-r_{-}^{2}}{r_{+}}\right), \quad \Omega=\frac{1}{L} \frac{r_{-}}{r_{+}} .
\end{aligned}
$$

The 2D CFT dual to the rotating BTZ black hole, although characterized by the same central charge (5), has different $L_{0}$ Virasoro operators for the right and left movers. The eigenvalues of these operators corresponding to a BTZ black hole of mass $M$ and angular momentum $J$ are $L_{0}=1 / 2(M L+J), \bar{L}_{0}=1 / 2(M L-J)$. The thermal density matrix for the CFT is given by $\rho=\exp (-\beta H+\beta \Omega P)$, where $H$ and $P$ are the Hamiltonian and the momentum operators. In the canonical description of the thermal $2 \mathrm{D}$ CFT this amounts to consider two different inverse temperatures

$$
\beta_{ \pm}=\beta(1 \pm \Omega)=2 \pi L^{2}\left(r_{+} \pm r_{-}\right)^{-1}
$$

for the right and left oscillators respectively. The entanglement entropy for the thermal $2 \mathrm{D}$ CFT in the cylinder $C$ and for a spacelike slice of length $\gamma$ is now given by [39]

$$
S_{\text {ent }}^{C F T}=\frac{c}{6} \ln \left[\frac{\beta_{+} \beta_{+}}{\pi^{2} \epsilon^{2}} \sinh \frac{\pi \gamma}{\beta_{+}} \sinh \frac{\pi \gamma}{\beta_{-}}\right] .
$$

The length $\gamma$ can be determined in the same way as for the spinless BTZ black hole. For the thermal CFT with two different temperatures for right and left movers we have the energy/temperature relation

$$
E_{R}+E_{L}-E_{0 R}-E_{0 L}=\frac{c}{12} \pi \gamma\left(T_{+}^{2}+T_{-}^{2}\right)
$$

Using Eq. (37) into Eq. (39) and comparing it with the black hole mass (35), we obtain easily $\gamma=2 \pi L$. As for the spinless case, we renormalize the entanglement entropy by subtracting the contribution to the vacuum coming from the left and right movers $S_{v a c}^{e n t}=c / 6(\ln (2 \pi L / \epsilon)+\ln (2 \pi L / \epsilon))$. Putting all together, we get the renormalized entropy

$$
\tilde{S}_{B T Z}^{e n t}=\frac{L}{4 G_{3}} \ln \left[\frac{L^{2}}{\pi^{2}\left(r_{+}+r_{-}\right)\left(r_{+}-r_{-}\right)} \sinh \frac{\pi\left(r_{+}+r_{-}\right)}{L} \sinh \frac{\pi\left(r_{+}-r_{-}\right)}{L}\right] .
$$

Expanding the previous expression for $r_{+} \gg L$ and $r_{+} \gg r_{-}$we get

$$
\tilde{S}_{B T Z}^{e n t}=\frac{\pi}{2 G_{3}} r_{+}-\frac{L}{2 G_{3}} \ln \frac{\pi r_{+}}{2 G_{3}}+O(1)=S_{B H}-\frac{L}{2 G_{3}} \ln S_{B H}+O(1) .
$$

\section{HOLOGRAPHIC ENTANGLEMENT ENTROPY OF CONICAL SINGULARITIES}

Let us now consider the classical solution of 3D AdS gravity given by Eq. (6), which describes 3D AdS spacetime with conical singularities. As explained in Sect. IV, solution (6) can be locally obtained applying a diffeomorphism to the AdS spacetime (10). This transformation is the "spacelike" counterpart of "thermalization" mapping the metric (10) into the BTZ black hole. On the 2D conformal boundary of the 3D AdS spacetime this transformation is described by the map

$$
z=\exp \frac{(t-i \phi)}{\beta}
$$


where $\beta$ is easily determined by first applying the transformation (20) mapping full $\mathrm{AdS}_{3}$ into (10) and then using the rescaling (11): $\beta=\beta_{\text {con }}$, where $\beta_{\text {con }}$ is given by Eq. (13). In the limit $\beta_{\text {con }} \gg 2 \pi L$ (i.e. $L \gg r_{+}$) the map (42) corresponds to a plane/cylinder transformation that maps the CFT on the plane $\mathcal{P}$ on the CFT on the cylinder $\mathcal{C}$. Thus, this conformal transformation maps the EE of a CFT on the plane $\mathcal{P}$ in the EE of a CFT in the cylinder $\mathcal{C}$ [28], i.e. the EE of a CFT at zero temperature and noncompact spacelike dimension given by Eq. (2) into the EE of a CFT at zero temperature with a compact spacelike dimension given by Eq. (11). Correspondingly, the holographic $\mathrm{EE}$ of the regularized AdS spacetime becomes the holographic EE associated to $\mathrm{AdS}_{3}$ with a conical singularity

$$
S_{\text {ent }}^{c o n}=\frac{c}{3} \ln \frac{\beta_{\text {con }}}{\pi \epsilon} \sin \frac{2 \pi^{2} L}{\beta_{\text {con }}}=\frac{c}{3} \ln \frac{2 L^{2}}{r_{+} \epsilon} \sin \frac{\pi r_{+}}{L} .
$$

Eq. (43) can be considered as the analytic continuation $r_{+} \rightarrow i r_{+}$of Eq. (29). The holographic entanglement entropy of a conical singularity described by a deficit angle $2 \pi\left(1-2 \pi L / \beta_{\text {con }}\right)$ is the analytic continuation of the holographic EE for the BTZ black hole with inverse temperature $\beta_{H}=\beta_{\text {con }}$. The analytic continuation corresponds to the exchange of the (compact) timelike with the spacelike direction. This result is a consequence of the modular symmetry (24) of the boundary CFT on the torus relating the BTZ solution and the conical singularity spacetime. In the limit $r_{+} \gg L$ the boundary torus corresponding to the BTZ black hole can be approximated by the infinitely long (along the spacelike direction) cylinder $C$. The modular transformation (24) maps the cylinder $C$ into the cylinder $\mathcal{C}$, which has infinitely long direction along the timelike direction and approximates the torus for $L \gg r_{+}$. Correspondingly the EE for the BTZ black hole (29) is transformed in the EE for the conical singularity (43).

\section{ENTANGLEMENT ENTROPY OF THE BTZ BLACK HOLE AND THERMAL ENTROPY OF CFT ON THE TORUS}

In the previous sections we have discussed the holographic EE of gravitational configurations in 3D AdS spacetime. In our approach the entanglement entropy of the boundary CFT, $S_{e n t}^{C F T}(\gamma, \beta)$, is used to probe thermal correlations at scales set by $\beta$ and spatial correlations at scales set by $\gamma$. The bulk description depends crucially on the regime of the $\mathrm{AdS}_{3} / \mathrm{CFT}_{2}$ correspondence we want to investigate.

First of all, we work in the region of validity of the gravity description of the AdS/CFT correspondence, when the AdS length is much larger than the Planck length,

$$
\frac{L}{G_{3}} \sim c \gg 1
$$

that is in the large $N$ approximation.

Moreover, considering curvature effects much smaller than the curvature of the AdS spacetime $1 / L^{2}$ allows the identification of the external parameter $\gamma$ in terms of $L$. On the other hand, the thermal scale $\beta$ can be easily identified, when a black hole is present in the bulk: $\beta=\beta_{H}=1 / T_{H}$. The semiclassical description for black holes holds when the horizon radius is much larger than the Planck length, $r_{+} \gg G_{3}$, whereas the holographic EE formula (30) holds for $r_{+} \gg L$. We are in the regime where we are allowed to approximate the boundary torus with the cylinder $C$. The path integral of Euclidean quantum gravity on $\mathrm{AdS}_{3}$ is dominated by the contribution coming from the BTZ black hole at $T=T_{H}$. The leading term in the EE (31) describes the main (thermal) contribution of the BTZ geometry and corresponds to the entanglement entropy for the CFT dominated completely by thermal correlations. When we increase the energy scale, we reach a regime for which contributions coming from geometries different from the BTZ instanton cannot be neglected. Quantum entanglement and the subleading term in Eq. (31) become relevant.

The other regime we have investigated so far is $L \gg r_{+}$, which is related to the previous one by the modular transformation (24). The Euclidean quantum gravity partition function for 3D AdS gravity is now dominated by $\mathrm{AdS}_{3}$ at temperature $T_{H}$. Although the solutions (6) describe singular geometries with conical singularities - therefore they cannot be part of the physical spectrum of the theory - the modular symmetry strongly indicates that they can be used to probe quantum entanglement. In this regime the boundary torus can be described by the cylinder $\mathcal{C}$ and the EE is given by Eq. (43).

One may now wonder about the regime $r_{+} \sim L$. In this parameter region we cannot approximate the torus with an infinitely long cylinder. $r_{+}=L$ is the fixed point of the modular transformations (22), (24) and we have a large $N$ phase transition, which is the 3D analogue of the Hawking-Page transition [26]. Because now the dual boundary CFT lives in the torus $\mathcal{T}\left(\beta_{H}, 2 \pi L\right)$, our calculations of the EE on the cylinder loose their validity. Furthermore, it is not a priori evident that the very notion of $\mathrm{EE}$ would maintain a sensible physical meaning in a regime where the semiclassical description of gravity is expected to fail.

The most direct way to learn something about the relationship between the two regimes $r_{+} \sim L$ and $r_{+} \gg L$ is to compare the $L \gg \beta$ asymptotic behavior of the thermal entropy $S_{t h}(\beta, L)$, derived from the partition function of the 
dual CFT on the torus, with the EE given by Eq. (30). Unfortunately, whereas the EE for a 2D CFT on a cylinder has an universal form, the thermal entropy $S_{t h}(\beta, L)$ for the CFT on the torus takes different form depending on the details of the CFT we are dealing with [56].

Here we will use a simple, albeit not completely general, approach to this problem. We will show that for the most common 2D CFTs (free bosons, free fermions, minimal models and Wess-Zumino-Witten models) the asymptotic, large temperature $L \gg \beta$ behavior of $S_{t h}(\beta, L)$ calculated from the partition function of the CFT on the torus reproduces exactly the leading term of the EE (30) for the BTZ black hole.

The partition function of the CFT on the torus, $Z(\tau)$, is a function of the modular parameter $\tau=i \beta / 2 \pi L$. Moreover, we will make use of the modular invariance of the partition function under the modular transformation (15) to write $Z(\tau)=Z(-1 / \tau)$. From the partition function one can easily compute the thermal entropy

$$
S_{t h}=\log Z-\beta \partial_{\beta}(\log Z) .
$$

We are interested in the asymptotic expansion of $S_{t h}$ in terms of the variable

$$
y=\sinh \left(\frac{2 \pi^{2} L}{\beta}\right)
$$

when $y \rightarrow \infty$. The asymptotic form of $S_{t h}^{(a s)}(y)$ is determined by first writing $Z$ as a function of the usual variable $q=\exp (2 \pi i \tau)$. After making use of the modular invariance of the partition function under the modular transformation (15), we will introduce the new variable $\tilde{q}=q(-1 / \tau)=\exp (-2 \pi i / \tau)$ and determine the $\tilde{q} \rightarrow 0$ asymptotic expansion of $Z(\tilde{q})$. Finally, we will determine $S_{t h}^{a s}(y)$ by making use of the $y \rightarrow \infty$ asymptotic expansion

$$
\tilde{q}=\frac{1}{4 y^{2}}+\mathcal{O}\left(\frac{1}{y^{4}}\right)
$$

Let us sketch the results of our calculations for the four cases under consideration.

Free bosons

The partition function for free bosons on the torus is [52]

$$
Z(\tau)=(\operatorname{Im} \tau)^{-\frac{c}{2}}|\eta(\tau)|^{-2 c},
$$

where $\eta$ is the Dedekind function

$$
\eta(\tau)=q^{\frac{1}{24}} \prod_{n=1}^{\infty}\left(1-q^{n}\right) .
$$

The $\tilde{q} \rightarrow 0$ asymptotic expansion for the entropy turns out to be

$$
S_{t h}(\tilde{q})=-\frac{c}{6} \ln \tilde{q}+\mathcal{O}(\ln (-\ln \tilde{q})),
$$

whereas the $y \rightarrow \infty$ expansion is

$$
S_{t h}(y)=\frac{c}{3} \log y+\mathcal{O}(\ln (\ln y)) .
$$

Free fermions

The partition function for free fermions on the torus is [52]

$$
Z(\tau)=\sum_{i=2}^{4}\left|\frac{\theta_{i}(\tau)}{\eta(\tau)}\right|^{2 c},
$$

where we have introduced the modular functions:

$$
\begin{aligned}
& \theta_{2}(\tau)=2 q^{\frac{1}{8}} \prod_{n=1}^{\infty}\left(1-q^{n}\right)\left(1+q^{n}\right)^{2} \\
& \theta_{3}(\tau)=\prod_{n=1}^{\infty}\left(1-q^{n}\right)\left(1+q^{n-\frac{1}{2}}\right)^{2} \\
& \theta_{4}(\tau)=\prod_{n=1}^{\infty}\left(1-q^{n}\right)\left(1-q^{n-\frac{1}{2}}\right)^{2}
\end{aligned}
$$


In this case we find the $\tilde{q} \rightarrow 0$ and $y \rightarrow \infty$ asymptotic expansion for the entropy

$$
S_{t h}=-\frac{c}{6} \ln \tilde{q}+\mathcal{O}(1)=\frac{c}{3} \ln y+\mathcal{O}(1)
$$

Minimal models

The partition function now is given by [52]

$$
Z(\tau)=\sum_{h, \bar{h}} \chi_{h}(\tau) \mathcal{M}_{h, \bar{h}} \bar{\chi}_{\bar{h}}(\bar{\tau})
$$

where $\chi_{h}$ are the Virasoro characters:

$$
\chi_{h}(\tau)=\frac{q^{h-\frac{c-1}{24}}}{\eta(q)}
$$

and $\mathcal{M}_{h, \bar{h}}$ are the so-called mass matrix elements. Using the asymptotic form for the Euler $\varphi$-function,

$$
\varphi(\tilde{q})=(1-\tilde{q})\left[1+\mathcal{O}\left(\tilde{q}^{2}\right)\right]
$$

one finds that the partition function has the following $\tilde{q} \rightarrow 0$ asymptotic expansion

$$
Z=A \tilde{q}^{-\frac{c}{12}}(1-\tilde{q})^{\alpha}\left[1+\mathcal{O}\left(\tilde{q}^{2}\right)\right]
$$

where $A=\mathcal{M}_{0,0}, \alpha=-2(d+1)$ and $d=\mathcal{M}_{1,0} / \mathcal{M}_{0,0}$.

Using Eqs. (45) and (46) one finds also in this case for the entropy the same asymptotic form given in Eq. (50).

Wess-Zumino-Witten models

The partition function for Wess-Zumino-Witten models is [52]

$$
Z(\tau)=\sum_{\hat{\lambda}, \hat{\xi}} \chi_{\hat{\lambda}}(\tau) \mathcal{M}_{\hat{\lambda}, \hat{\xi}} \bar{\chi}_{\hat{\xi}}(\bar{\tau})
$$

with the characters $\chi_{\hat{\lambda}}$ given by:

$$
\chi_{\hat{\lambda}}(\tau) \equiv \chi_{\lambda_{1}}^{(k)}(\tau)=\frac{q^{\frac{\left(\lambda_{1}+1\right)^{2}}{4(k+2)}}}{[\eta(q)]^{3}} \sum_{n \in Z}\left[\lambda_{1}+1+2 n(k+2)\right] q^{n\left[\lambda_{1}+1+(k+2) n\right]} .
$$

The asymptotic expansion for the characters is

$$
\chi_{\lambda_{1}}^{(k)}(-1 / \tau)=\frac{\tilde{q}^{\frac{\left(\lambda_{1}+1\right)^{2}}{4(k+2)}-\frac{1}{8}}}{[\varphi(\tilde{q})]^{3}}\left[\lambda_{1}+1+\mathcal{O}\left(\tilde{q}^{k-\lambda_{1}+1}\right)\right],
$$

from which it follows the $\tilde{q} \rightarrow 0$ asymptotic form of the partition function:

$$
\begin{aligned}
Z= & {[\varphi(\tilde{q})]^{-3} \sum_{\lambda_{1}, \mu_{1}} \mathcal{M}_{\lambda_{1}, \mu_{1}} \tilde{q}^{\frac{\left(\lambda_{1}+1\right)^{2}+\left(\mu_{1}+1\right)^{2}}{4(k+2)}-\frac{1}{4}} \times } \\
& {\left[\lambda_{1}+1+\mathcal{O}\left(\tilde{q}^{k-\lambda_{1}+1}\right)\right] \times\left[\mu_{1}+1+\mathcal{O}\left(\tilde{q}^{k-\mu_{1}+1}\right)\right] . }
\end{aligned}
$$

The leading terms in the previous summation are those with $\lambda_{1}=\mu_{1}=0$, so that, using the asymptotic form (51) for the $\varphi$-function, one finds

$$
Z=\mathcal{M}_{0,0} \tilde{q}^{-\frac{k}{4(k+2)}}(1-\tilde{q})^{-3}\left[1+\mathcal{O}\left(\tilde{q}^{2}\right)\right]
$$

Specializing to the case of an $s u(2)_{k}$ algebra, for which one has $c=\frac{3 k}{k+2}$, Eq. (53) takes the form given by Eq. (52) with $A=\mathcal{M}_{0,0}$ and $\alpha=-3$. Both $A$ and $\alpha$ do not enter in the leading term of the asymptotic expansion for the entropy, but determine only the subleading terms. Thus, making use of Eqs. (45) and (46), one finds also in this case for the entropy the same asymptotic form given in Eq. (50). 


\section{FINAL REMARKS}

Let us first summarize the main result of the previous section. The leading term in the large temperature, $y \rightarrow \infty$ expansion of the thermal entropy for the four CFT classes on the torus considered in this paper,

$$
S_{t h} \sim \frac{c}{3} \ln y=\frac{c}{3} \ln \sinh \frac{2 \pi^{2} L}{\beta},
$$

reproduces for $\beta=\beta_{H}$ the leading term of the holographic EE for the BTZ black hole given by Eq. (30). This result sheds light on the meaning of the holographic EE for the BTZ black hole in particular and, more in general, on the very meaning of entanglement for black holes. In fact our result indicates that entanglement entropy for black hole is a semiclassical concept that has a meaning only for macroscopical black holes in the regime $r_{+} \gg L$. Thus, entanglement seems to arise from a purely thermal description of the underlying quantum theory of gravity which is assumed to describe 3D quantum gravity in the region $r_{+} \sim L$. This fact supports the point of view that the microscopic theory describing the BTZ black hole at short scales is unitary. Entanglement entropy is an emergent concept, which comes out when the semiclassical notion of spacetime geometry is used to describe the black hole. The agreement between thermal entropy for the CFT on the torus and holographic EE for the BTZ black hole is limited to the leading term in the $y \rightarrow \infty$ expansion. The subleading terms in the expansions (49) and (50) are not of the same order for the different CFT we have considered. The subleading terms are of order $\ln (\ln y)$ for the free boson, whereas they are $O(1)$ for the other three cases. These subleading terms seem to be not universal but they depend on the actual CFT we are dealing with.

An other important point, which we have only partially addressed in this paper, concerns the role played by the classical solutions of 3D AdS gravity describing conical singularities of the spacetime. Because they represent singular geometries, they cannot be part of the physical spectrum of pure 3D AdS gravity (although they may play a role for gravity interacting with pointlike matter). On the other hand, they are related with the BTZ black hole solutions by modular transformations and one can associate to them an entanglement entropy. All this could be very useful for shedding light on the phase transition (analogue to the Hawking-Page transition of four-dimensional gravity), which is expected to take place at $r_{+}=L$.

[1] A. Strominger and C. Vafa, Phys. Lett. B 379 (1996) 99 arXiv:hep-th/9601029.

[2] G. T. Horowitz and A. Strominger, Phys. Rev. Lett. 77 (1996) 2368 arXiv:hep-th/9602051.

[3] A. Strominger, JHEP 9802 (1998) 009 arXiv:hep-th/9712251.

[4] M. Cadoni and S. Mignemi, Phys. Rev. D 59 (1999) 081501 arXiv:hep-th/9810251.

[5] S. Carlip, Phys. Rev. Lett. 82 (1999) 2828 arXiv:hep-th/9812013.

[6] S. Carlip, Phys. Rev. Lett. 88 (2002) 241301 arXiv:gr-qc/0203001].

[7] S. Carlip, J. Phys. Conf. Ser. 33 (2006) 73.

[8] S. Carlip, Gen. Rel. Grav. 39 (2007) 1519 arXiv:0705.3024 [gr-qc]].

[9] A. Strominger, arXiv:0906.1313 [hep-th].

[10] G. Vidal, J. I. Latorre, E. Rico and A. Kitaev, Phys. Rev. Lett. 90 (2003) 227902 arXiv:quant-ph/0211074].

[11] A. Kitaev and J. Preskill, Phys. Rev. Lett. 96 (2006) 110404 arXiv:hep-th/0510092.

[12] J. I. Latorre, C. A. Lutken, E. Rico and G. Vidal, flows," Phys. Rev. A 71 (2005) 034301 arXiv:quant-ph/0404120.

[13] V. E. Korepin, Phys. Rev. Lett. 92 (2003) 964021.

[14] H. Casini and M. Huerta, Phys. Lett. B 600 (2004) 142 arXiv:hep-th/0405111.

[15] D. V. Fursaev, of quantum Phys. Rev. D 73 (2006) 124025 arXiv:hep-th/0602134.

[16] M. Fujita, T. Nishioka and T. Takayanagi, arXiv:0806.3118 [hep-th].

[17] H. Casini and M. Huerta, arXiv:0905.2562 [hep-th].

[18] G. 't Hooft, Nucl. Phys. B 256 (1985) 727.

[19] V. P. Frolov and I. Novikov, Phys. Rev. D 48 (1993) 4545 arXiv:gr-qc/9309001.

[20] R. B. Mann and S. N. Solodukhin, Phys. Rev. D 55 (1997) 3622 arXiv:hep-th/9609085].

[21] D. V. Fursaev, arXiv:0711.1221 [hep-th].

[22] T. M. Fiola, J. Preskill, A. Strominger and S. P. Trivedi, Phys. Rev. D 50 (1994) 3987 arXiv:hep-th/9403137.

[23] V. P. Frolov, D. V. Fursaev and A. I. Zelnikov, Nucl. Phys. B 486 (1997) 339 arXiv:hep-th/9607104.

[24] M. Cadoni, Phys. Lett. B 653 (2007) 434 arXiv:0704.0140 [hep-th]].

[25] M. Cadoni, PoS QG-PH (2007) 013 arXiv:0709.0163 [hep-th]].

[26] O. Aharony, S. S. Gubser, J. M. Maldacena, H. Ooguri and Y. Oz, Phys. Rept. 323 (2000) 183 arXiv:hep-th/9905111.

[27] C. Holzhey, F. Larsen and F. Wilczek, Nucl. Phys. B 424 (1994) 443 arXiv:hep-th/9403108.

[28] P. Calabrese and J. L. Cardy, J. Stat. Mech. 0406 (2004) P002 arXiv:hep-th/0405152.

[29] P. Calabrese and J. Cardy, arXiv:0905.4013 [cond-mat.stat-mech]. 
[30] L. Susskind and E. Witten, arXiv:hep-th/9805114

[31] A. W. Peet and J. Polchinski, Phys. Rev. D 59 (1999) 065011 arXiv:hep-th/9809022.

[32] R. Emparan, JHEP 0606 (2006) 012 arXiv:hep-th/0603081.

[33] S. N. Solodukhin, Phys. Rev. Lett. 97 (2006) 201601 arXiv:hep-th/0606205].

[34] S. Hawking, J. M. Maldacena and A. Strominger, JHEP 0105, 001 (2001) arXiv:hep-th/0002145.

[35] T. Azeyanagi, T. Nishioka and T. Takayanagi, Phys. Rev. D 77 (2008) 064005 [arXiv:0710.2956 [hep-th]].

[36] S. Ryu and T. Takayanagi, Phys. Rev. Lett. 96 (2006) 181602 arXiv:hep-th/0603001.

[37] S. Ryu and T. Takayanagi, JHEP 0608 (2006) 045 arXiv:hep-th/0605073.

[38] D. V. Fursaev, JHEP 0609 (2006) 018 arXiv:hep-th/0606184.

[39] V. E. Hubeny, M. Rangamani and T. Takayanagi, JHEP 0707 (2007) 062 arXiv:0705.0016 [hep-th]].

[40] G. Michalogiorgakis, arXiv:0806.2661 [hep-th].

[41] J. R. Sun, arXiv:0810.0967 [hep-th].

[42] T. Nishioka, S. Ryu and T. Takayanagi, arXiv:0905.0932 [hep-th].

[43] S. Carlip, Class. Quant. Grav. 22 (2005) R85 arXiv:gr-qc/0503022.

[44] E. Witten, arXiv:0706.3359 [hep-th].

[45] A. Maloney and E. Witten, arXiv:0712.0155 [hep-th].

[46] J. D. Brown and M. Henneaux, Commun. Math. Phys. 104 (1986) 207.

[47] M. Banados, C. Teitelboim and J. Zanelli, Phys. Rev. Lett. 69 (1992) 1849 arXiv:hep-th/9204099.

[48] M. Banados, M. Henneaux, C. Teitelboim and J. Zanelli, Phys. Rev. D 48 (1993) 1506 arXiv:gr-qc/9302012].

[49] S. Carlip and C. Teitelboim, Phys. Rev. D 51 (1995) 622 arXiv:gr-qc/9405070.

[50] S. W. Hawking and D. N. Page, Commun. Math. Phys. 87, 577 (1983).

[51] Y. Kurita and M. a. Sakagami, Prog. Theor. Phys. 113 (2005) 1193 arXiv:hep-th/0403091.

[52] P. Di Francesco, P. Mathieu and D. Senechal, New York, USA: Springer (1997) $890 p$

[53] A remarkable exception is represented by 2D AdS gravity. In 2D black hole entropy can be ascribed to quantum entanglement if Newton constant is wholly induced by quantum fluctuations [22, 23]. This fact allows a simple derivation of the EE of 2D AdS black hole [24, 25].

[54] The length-scales $\gamma$ and $\epsilon$ are defined up to a dimensionless multiplicative constant of $O(1)$. In the following we will set this multiplicative constant equal to $2 \pi$.

[55] Because the authors of Ref. [20] consider the case of a single scalar field, to reproduce their result we obviously need to use for the central charge $c+\bar{c}=1$

[56] Despite the intense activity on the subject in the last decade, the exact form of the 2D CFT dual to pure 3D AdS gravity remains still a controversial point [43, 44, 45]. 